\title{
The EIT coil-like structure in the March 20, 2000 eruptive prominence
}

\author{
M. Karlický and S. Šimberová \\ Astronomical Institute, Academy of Sciences, 25165 Ondřejov, Czech Republic \\ Received 9 August 2001 / Accepted 18 February 2002

\begin{abstract}
The evolution of the March 20, 2000 prominence eruption observed in the $195 \AA$ (EIT/SOHO), H $\alpha$, (MDI/SOHO), 1-3 GHz radio and hard X-ray (BATSE) was analyzed. A unique EIT coil-like structure with a hot core, indicating an electric current, was observed at the beginning of the prominence eruption. This structure with about 5 turns was associated with the reverse drift radio bursts at $1.0-2.5 \mathrm{GHz}$ and the $33-57 \mathrm{keV}$ hard X-ray emission. The hot core observation confirmed our recent suggestion about heating inside the eruptive prominence. Comparing the form of the EIT filament in the pre-eruption phase with the magnetic field map the presence of the electric current was confirmed. It was suggested that the relatively slow drifts of the reverse drift bursts can be due to a beam propagating in a vertical coil-like (helical) structure. Plasma processes and parameters in the eruptive prominence were discussed.
\end{abstract}

Key words. Sun: activity - Sun: prominences - Sun: magnetic field

\section{Introduction}

The close connection of eruptive prominences to the coronal mass ejection (CME) has led to an increasing interest in them (Schmieder et al. 1997; Wang \& Sheeley 1999; Wood et al. 1999; Ciaravella et al. 2000; Hori 2000). Formation, structure, evolution and stability of eruptive prominences have been studied in many papers (Tandberg-Hanssen 1974; Schmahl \& Hildner 1977; Rompolt 1978, 1990; Ballester \& Kleczek 1983, 1984; Moore 1988; Vršnak et al. 1991). In traditional studies authors mainly tried to explain how the cool and dense prominence material is sustained. Now, in connection with CMEs, the principal question is: what are the causes and initial stages of the prominence eruption? The most probable processes are those connected with the prominence magnetic rope (Vršnak et al. 1991). Extended magnetic loops connected with the $\mathrm{H} \alpha$ prominences were found (Dere et al. 1999; Plunkett et al. 2000).

Several models of the CME including the eruptive prominence were suggested. Chen (1996) presented a model of prominence eruption based on an instability of the extended magnetic flux rope. In the Antiochos et al. (1999) model the reconnection removes the unsheared field above the low-lying, sheared core flux near the neutral line, thereby allowing this core flux to burst open.

Send offprint requests to: M. Karlický, e-mail: karlicky@sunkl.asu.cas.cz
Furthermore, Amari et al. (2000) presented the twisted flux rope model for the CMEs and two-ribbon flares.

Recently, the expansion, rotational and axial velocities in the May 15, 2000 eruptive prominence were derived from almost regular elliptical features observed in the $\mathrm{H} \alpha$ spectra (Karlický et al. 2001a). It agrees with the concept of an untwisting process of the magnetic rope during the prominence eruption. Based on spectral observations it was suggested that the core of the eruptive prominence is heated.

In this paper we continue our studies of eruptive prominences. First, observations of the March 20, 2000 eruptive prominence are shown. Then, interesting EIT structures, the magnetic field in the region of the EIT filament, and indications of the electric current are presented. Finally, these observations are discussed in comparison with previous results. (Definitions: the term magnetic rope is used here in the sense introduced by Parker 1974.)

\section{Observations}

The SOHO/EIT images (Delaboudinière et al. 1995) of the eruptive prominence are combined with the $\mathrm{H} \alpha$ images observed at Ondřejov Observatory. The Ondřejov radio data show the reverse drift bursts, the SOHO/MDI magnetograms are used for the analysis of the magnetic field topology and the BATSE hard X-ray data are added for comparison. 


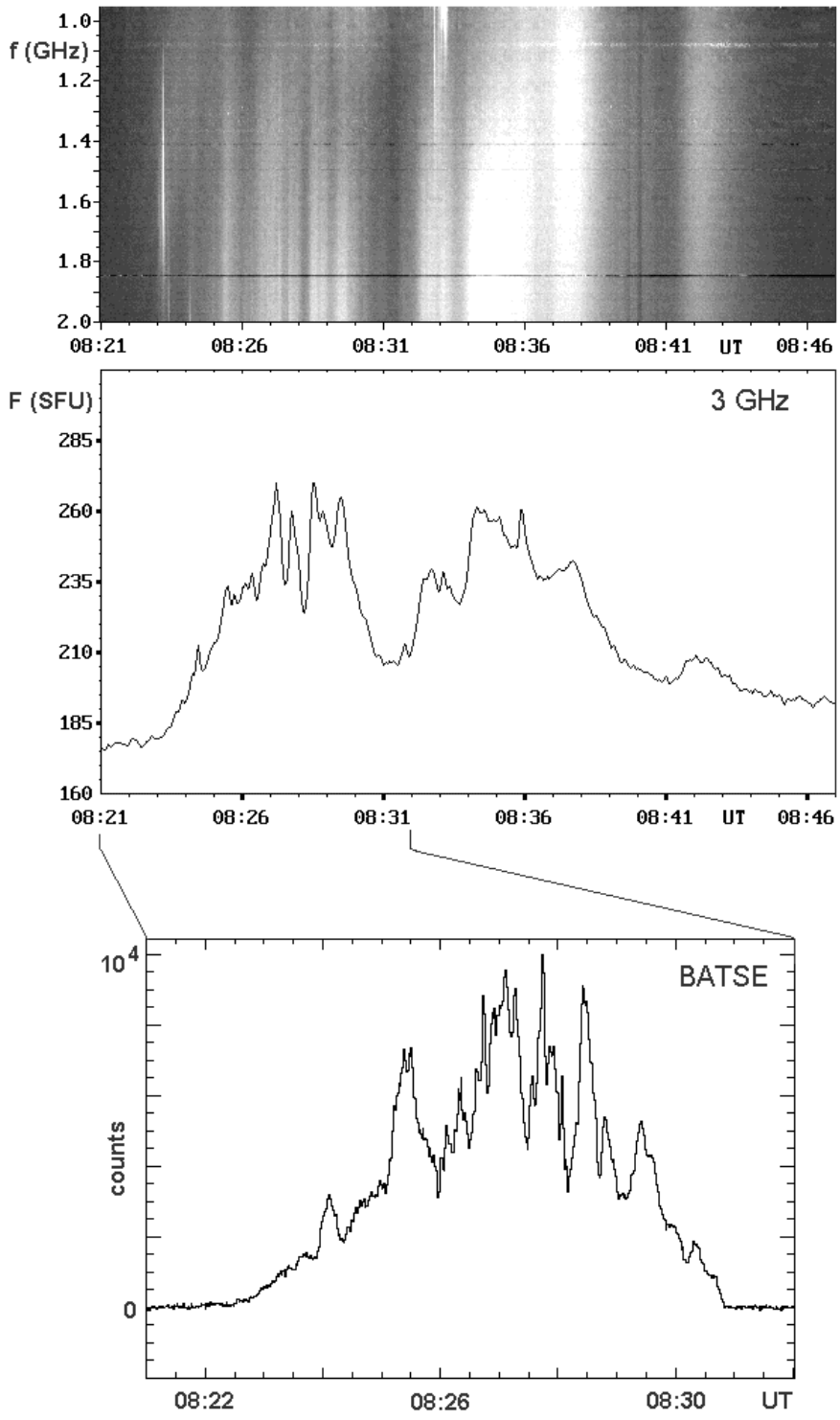

Fig. 1. The March 20, 2000 event. Upper: the 1-2 GHz Ondřejov radio spectrum, middle: the $3 \mathrm{GHz}$ Ondřejov radio flux, and bottom: the $33-57 \mathrm{keV}$ BATSE X-ray record.

The studied eruptive prominence was observed on March 20, 2000 in the active region NOAA AR8921 (S16E79) close to the south-east solar limb. It was accompanied by the solar flare M2.2/2B at 08:23 start max 08:34 - end 08:50 UT (NOAA Solar Events Report). The non-thermal emissions of this event are shown in Fig. 1, where the $1-2 \mathrm{GHz}$ radio spectrum, $3 \mathrm{GHz}$ radio and hard X-ray BATSE emissions are shown. Accordingly, the eruption processes started at 08:23 UT with broadband radio emission showing quasi-periodic oscillations with a characteristic period of about $30 \mathrm{~s}$. These oscillations can be seen also on the BATSE hard X-ray record. An enlargement of the $1-2$ and $2-4.5 \mathrm{GHz}$ radio spectra (Fig. 2) shows that the eruptive processes started with reverse drift bursts, which indicate an acceleration of electron beams and their propagation downwards in the solar atmosphere. These reverse drift bursts were observed

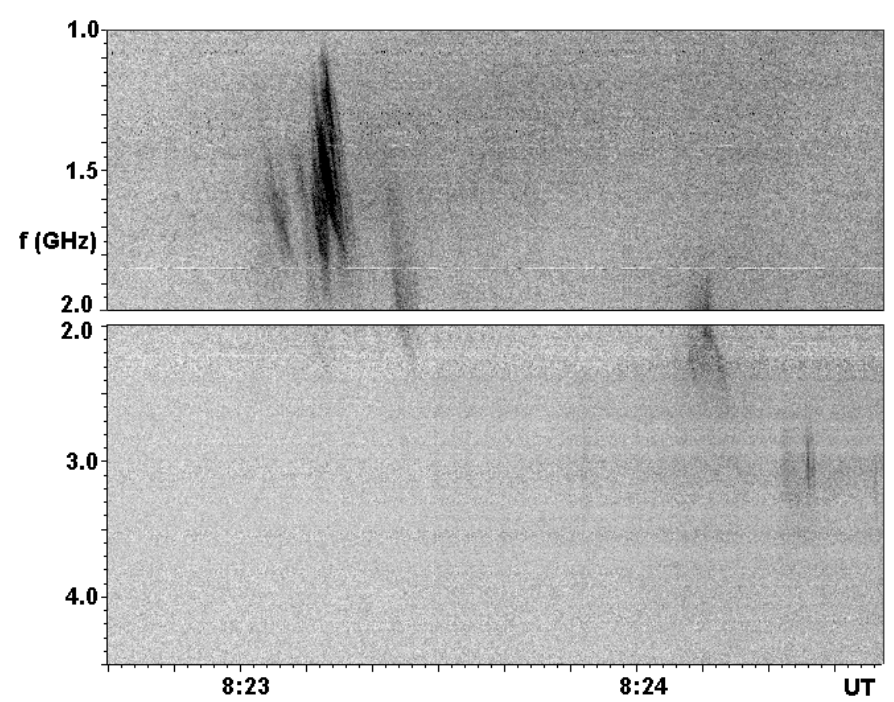

Fig. 2. The detailed Ondřejov radio spectra from two independent radiospectrographs in the $1-2 \mathrm{GHz}$ and $2-4.5 \mathrm{GHz}$ ranges with reverse drift bursts. Here the radio emission is expressed in black to increase a contrast of the radio spectra.

simultaneously with the observation of the EIT coil-like structure (see next paragraph) and associated with the 33-57 keV hard X-ray emission generated through the bremsstrahlung mechanism in deep atmospheric layers (see a time coincidence between the radio and hard $\mathrm{X}$ ray emissions in the $33-57 \mathrm{keV}$ range - Figs. 1 and 2). While the reverse drift bursts at 08:23:00-08:23:30 UT in the 1.05-2.3 GHz range correspond to the hard X-ray increase at 08:23:00-08:23:40 UT, the $1.8-2.5 \mathrm{GHz}$ reverse drift burst at 08:24:07-08:24:15 UT corresponds to the hard X-ray peak at 08:24:10 UT. The frequency drift of the reverse drift bursts is in the interval of $100-200 \mathrm{MHz} \mathrm{s}^{-1}$. As seen there is no simple relationship between intensity variations of the reverse drift bursts and hard X-ray emission. Namely, according to the theory, the intensity of the reverse drift burst is sensitive to the positive derivative of the distribution function of superthermal electrons in the velocity space and the intensity of the hard X-ray emission depends on the total electron flux (Benz 1993; Brown 1971). (Remark: The hard X-ray emission increase observed at 08:23-08:24 UT cannot be considered as a thermal one due to its high energy $(33-57 \mathrm{keV})$ ).

The evolution of the eruptive prominence in EIT $195 \AA$ and $\mathrm{H} \alpha$ is presented in Figs. $3-5$. As seen at 8:12 UT (Fig. 3 upper), i.e. before the eruption, the EIT image shows a dark filament. (Remark: In our previous studies it was found that the $\mathrm{H} \alpha$ prominence features correspond to those visible as dark ones on EIT $195 \AA$ images.) Then, at 8:24 UT (Fig. 3 middle) this filament is ejected upwards. In its southern leg the coil-like structure with about 5 turns can be seen. Inside the coil-like structure there is a hot core. The length $L$, diameter $D$ and the pitch length $P$ of the coil-like structure are roughly estimated as $45000 \mathrm{~km}, 15000 \mathrm{~km}$ and $9000 \mathrm{~km}$, respectively (see details in Fig. 4; the pitch length is defined in the 

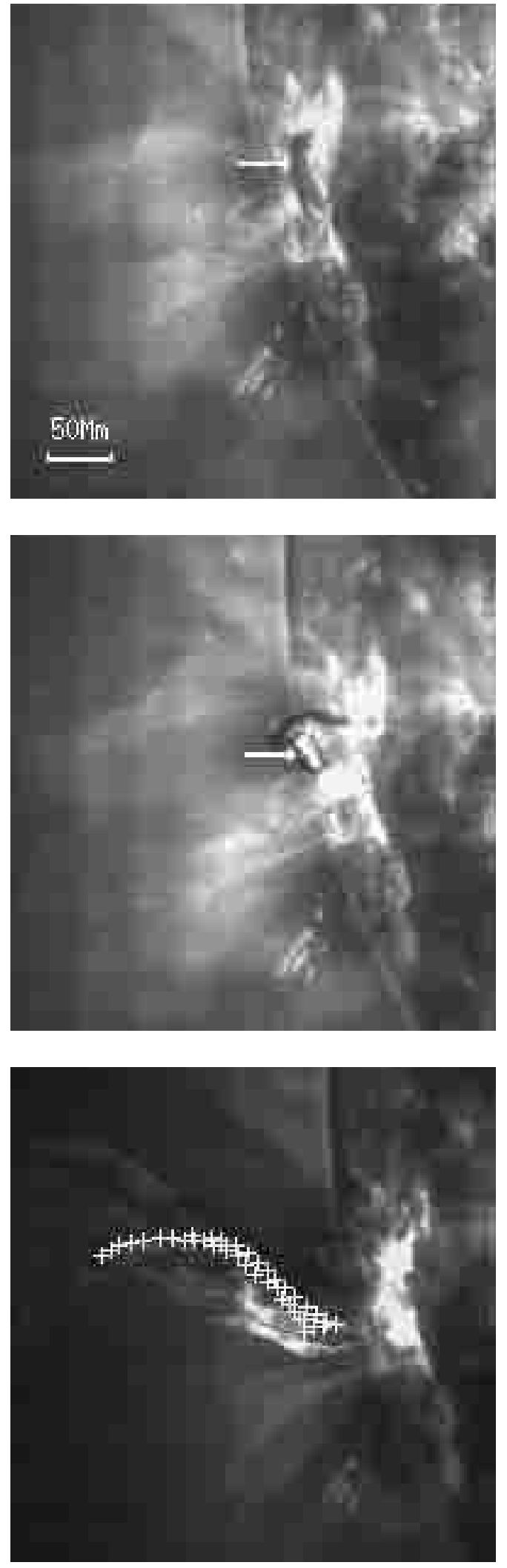

Fig. 3. The evolution of the eruptive prominence in the EIT observations (Fe XII, $195 \AA$ ) at 8:12 UT (upper), 8:24 UT (middle), and 8:36 UT (bottom), where the $\mathrm{H} \alpha$ eruptive prominence image observed at 8:35:48 UT is superimposed (the white crosses). The white arrows show the dark filament (upper) and the coil-like structure (middle).

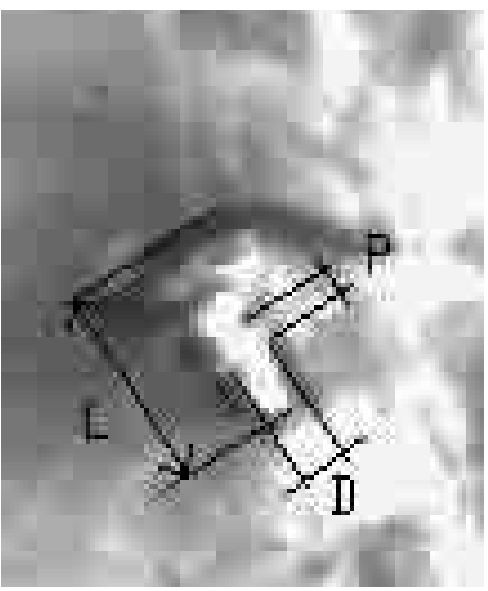

Fig. 4. The EIT coil-like structure observed at 8:24 UT. $L$ is the length, $D$ is the diameter, and $P$ is the pitch length of this structure.

same way as in paper by Vršnak et al. 1991). In the following time point (8:36 UT, Fig. 3 bottom) the eruption continues, the prominence plasma is heated and only a part remains cold, as revealed by the $\mathrm{H} \alpha$ observation (see the superposition of the $\mathrm{H} \alpha$ prominence in Fig. 3 bottom, and Fig. 5). In Fig. 6 the pre-eruption EIT filament (8:12 UT) is superimposed on the 8:15 UT MDI/SOHO magnetogram. The form of this EIT filament and its position in the magnetic field map indicate deviations of the magnetic field lines from the potential ones.

\section{Model of the slowly drifting reverse drift bursts}

Fast drifting bursts are observed in a very broad range of frequencies ( 3000-1 MHz) (Bastian et al. 1998; Suzuki \& Dulk 1985). These bursts are also called type III bursts. Absolute values of their frequency drifts decrease towards lower frequencies. In the frequency range below $\sim 1000 \mathrm{MHz}$ the frequency drift of type III bursts is usually negative. On the other hand, in the frequency range above $\sim 1000 \mathrm{MHz}$, in many cases the frequency drift of the fast drift bursts is positive (reverse drift bursts). Usually the drift of the positively drifting bursts is very high (greater than $1000 \mathrm{MHz} \mathrm{s}^{-1}$ ), which is why it is not possible to measure it using the standard radio spectra with a time resolution of only $0.1 \mathrm{~s}$. To determine higher frequency drifts, spectra with higher time resolution are necessary (see e.g. Karlický et al. 2001b). But in some cases, usually at the beginning of flares, the reverse drift bursts with slow frequency drifts are observed. The present event belongs to these cases. The bursts are observed in a limited frequency range and their frequency drift is about $100 \mathrm{MHz} \mathrm{s}^{-1}$. The statistics of these slowly drifting reverse drift bursts in solar flares is under preparation.

The type III and reverse drift bursts are explained by a so-called plasma emission mechanism (Benz 1993). The electron beams with velocities of $0.2-0.6 c$ ( $c$ is the speed of light) propagate through the coronal plasma, that has a density gradient. The beam generates the plasma 

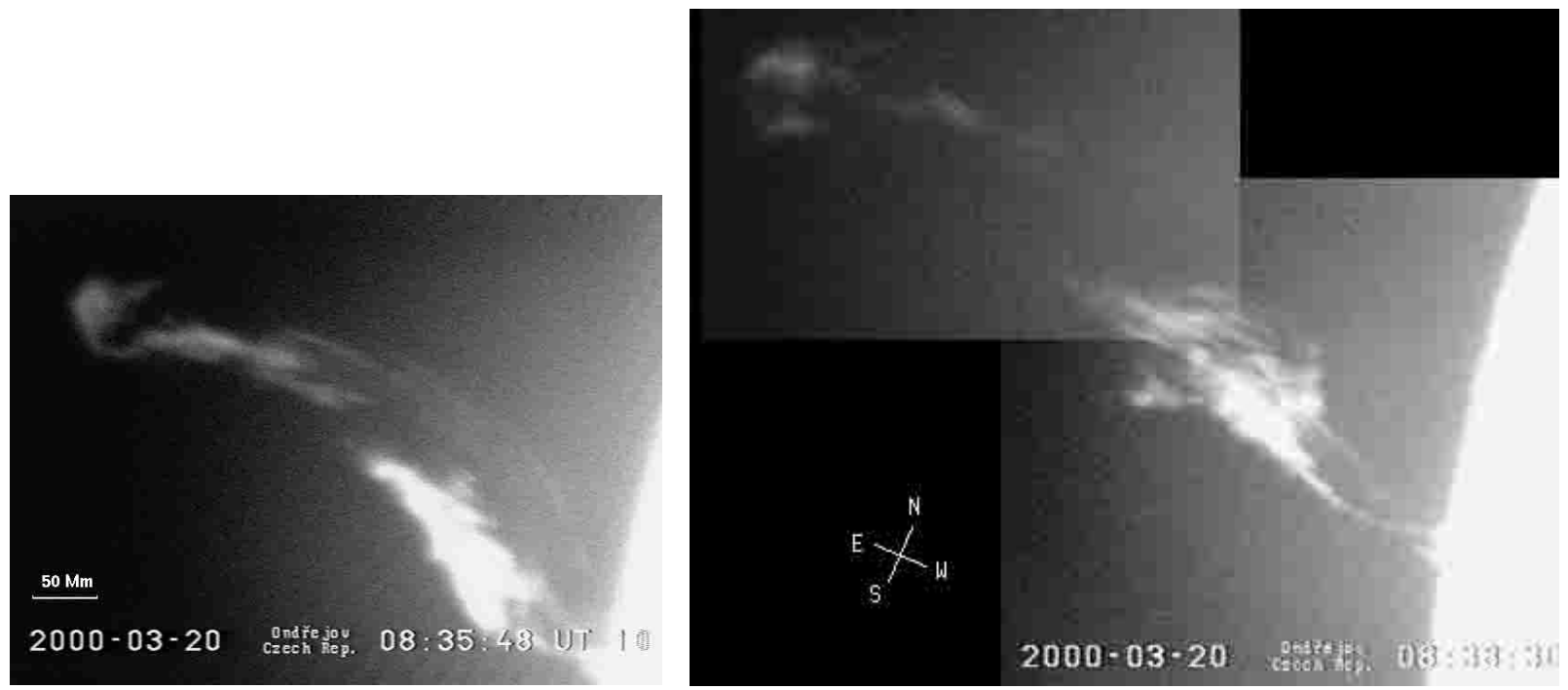

Fig. 5. The $\mathrm{H} \alpha$ images of the eruptive prominence: left) at 8:35:48 UT, and right) at 8:38:30 UT, observed at the Ondřejov Observatory. The north-south and east-west directions are shown in the middle of the figure.

waves on the local plasma frequency $\left(\omega^{2} \sim n_{\mathrm{e}}\right.$, where $n_{\mathrm{e}}$ is the electron plasma density) and these waves are then transformed into electromagnetic waves. The frequency of the electromagnetic (radio) waves is the same as the source plasma frequency (fundamental radio emission) or double this frequency (harmonic radio emission). Thus the electron beam, which propagates through the atmosphere with the density gradient, generates the radio burst drifting in frequencies. Due to gravity the density decreases in the vertical direction in the solar atmosphere. That is why the negatively drifting type III bursts are interpreted by beams propagating in an upwards direction and the reverse drift bursts by downwards propagating beams. Moreover, in the hydrostatic model of the solar atmosphere (e.g. Priest 1982) the density gradient is not constant; it decreases with the increase in height in the solar atmosphere. Thus, the absolute values of the frequency drifts decrease with the decrease in the frequency of the radio emission. Fast-drifting radio bursts in the $\mathrm{GHz}$ frequency range (frequency drifts greater than $1000 \mathrm{MHz} \mathrm{s}^{-1}$ ) are in accordance with this concept. But what about the reverse drift bursts with frequency drifts of $100-200 \mathrm{MHz} \mathrm{s}^{-1}$, also observed in our event? Beams with slower velocities can be suggested, but their velocities cannot be lower than about $0.2 c$ due to the Landau damping of plasma waves in the plasma model of these bursts. Slow proton beams were also suggested (Benz 1993). But in our case the bursts were observed simultaneously with the observation of the EIT coil-like structure and they were associated with the $33-57 \mathrm{keV}$ hard X-ray emission (the velocity of the $33 \mathrm{keV}$ electron is $0.36 \mathrm{c}$ ). Therefore we suggest here a model which agrees naturally with the observed coil-like structure; see the schema (Fig. 7). Namely, the electron beam propagates along the helical magnetic field line and it generates along its trajectory the radio burst. The frequency drift of this burst depends on the

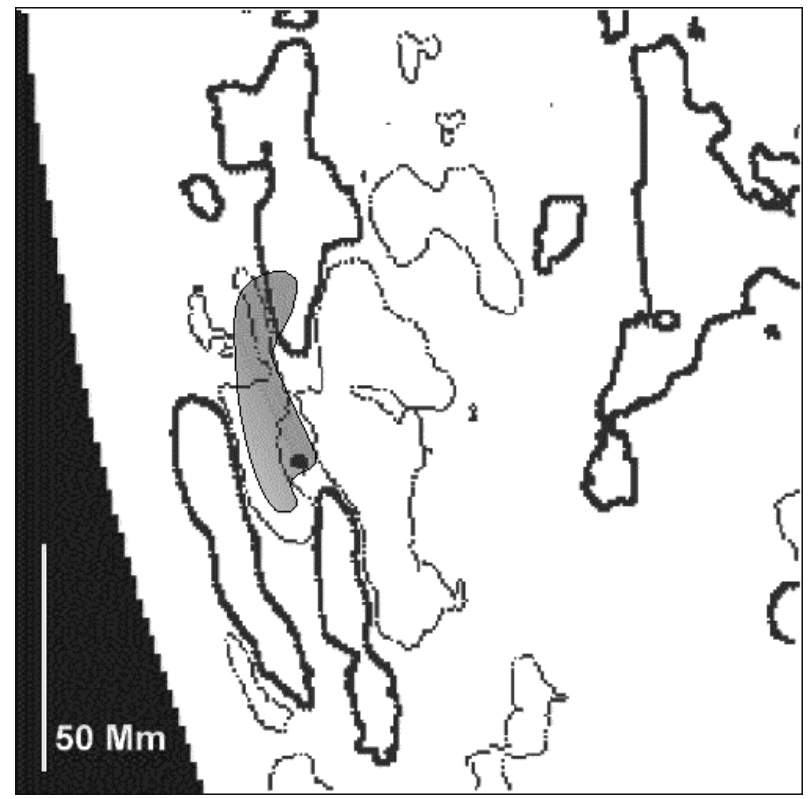

Fig. 6. The MDI/SOHO magnetic field observed at 8:15 UT with the EIT filament (shaded area) observed at 8:12 UT. Contours of the $+10 \mathrm{G}$ (thick black line) and $-10 \mathrm{G}$ (thin black line) magnetic field are drawn.

velocity component of the electron beam in the direction of the density gradient. In the usual circumstances, due to gravity, the density gradient is oriented in the vertical direction towards the solar photosphere. Thus, in the helical (coil-like) structure, having its axis nearly vertical, the angle between the direction of the beam propagation and the density gradient can be large. In this case the frequency drift of the generated burst is effectively reduced. 


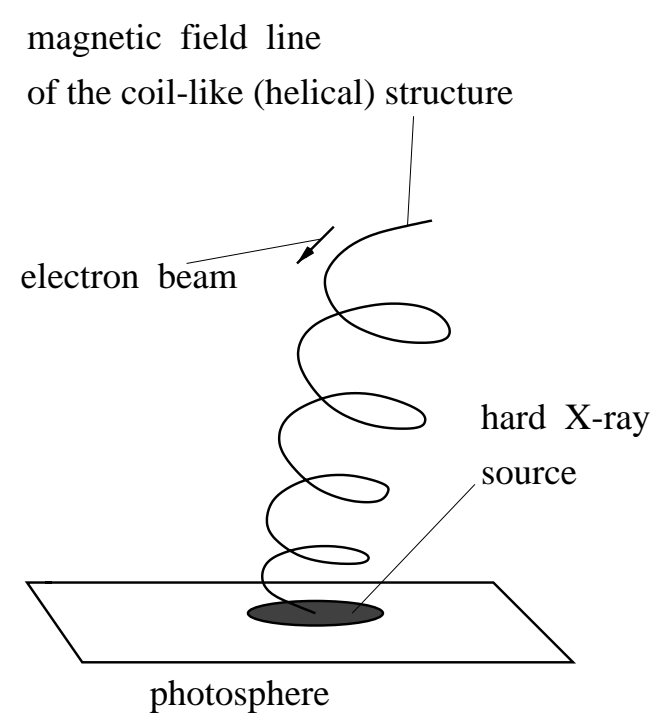

Fig. 7. The model of a helical trajectory of the electron beam in the solar atmosphere explaining slow frequency drifts of the reverse drift bursts.

\section{Discussion and conclusions}

In the southern leg of the EIT filament observed at 8:24 UT a unique coil-like structure with about 5 turns was observed. This structure indicates the electric current, which is non-neutralized within the coil-like structure (see Melrose 1991 for the classification of the coronal current-patterns). The presence of the electric current can be recognized also by comparing the pre-eruption EIT filament with the magnetic field map. Considering these facts and the magnetic field in the vicinity of the EIT filament, we interpret the pre-eruption EIT filament as a magnetic rope in the sense of the paper by Parker (1974). This interpretation is in agreement with the concept of a cylindrical prominence, which is due to the kink instability destabilized (Vršnak 1990). On the other hand, from the number of turns $(\sim 5)$ in the coil-like structure, the critical stability twist (for its definition, see Hood \& Priest 1979) of this cylindrical prominence can be estimated as $\sim 10 \pi$. These values of the twist correspond to those found for helical-like patterns in the phase of prominence acceleration (Vršnak et al. 1991).

The hot core of the coil-like structure is probably heated by the electric current flowing through this space. The existence of the hot core is in agreement with our recent spectroscopic observations. In the May 15, 2000 eruptive prominence almost regular elliptical features were observed in the $\mathrm{H} \alpha$ spectra (Karlický et al. 2001a), and the central parts of these features were, in the $\mathrm{H} \alpha$ emission, empty. We suggested that the central part of the eruptive prominence is heated. Now, if we take the electric current that flows through the coil-like structure (the diameter $15000 \mathrm{~km})$ as that estimated in prominences $\left(10^{10}-10^{12} \mathrm{~A}\right.$, Ballester \& Kleczek 1984, and references therein) then the mean current density in the heated core is in the range of $5.7 \times 10^{-5}-5.7 \times 10^{-3} \mathrm{~A} \mathrm{~m}^{-2}$. For comparison, the mean maximum current densities measured in the flaring active regions are about $10^{-2} \mathrm{~A} \mathrm{~m}^{-2}$ (Hagyard 1988). These values of current densities are generally low for a fast heating, therefore in the space of the heating we expect an electric current with a filamentary structure; the currents are concentrated in the current filaments.

Because the reverse drift bursts were observed simultaneously with the EIT coil-like structure, we used this structure in the model explaining the slow drifts of the bursts. Using this model for the fundamental radio emission in the frequency range of the reverse drift bursts $(1.05-2.5 \mathrm{GHz})$, the density in the coil-like structure can be estimated as $1.4 \times 10^{10} \mathrm{~cm}^{-3}-7.7 \times 10^{10} \mathrm{~cm}^{-3}$. These values are within the range of the typical electron densities of $10^{10} \mathrm{~cm}^{-3}-10^{11} \mathrm{~cm}^{-3}$ estimated in the prominences (Tandberg-Hanssen 1995).

It is interesting to note that after the period of observations of the slowly drifting reverse drift bursts (about $80 \mathrm{~s}$ ), broadband radio pulses without any measurable frequency drift were observed. This fact can be explained as follows: At the beginning of eruptive processes, individual beams accelerated at a localized acceleration space, propagate in an unperturbed coil-like structure and we observe them as reverse drift bursts. But later the acceleration space spreads into a much larger volume (probably also outside the coil-like structure) and many "beams" are accelerated. Then the radio spectrum will be a superposition of radio emissions of many "beams" and thus the resulting radio emission will be without any frequency drift. Moreover, due to the ohmic dissipation of the electric current, the original coil-like structure or its parts can be destroyed. In conclusion, if this model is real then the observations of the slow drifting reverse drift bursts at the beginning of some solar flares (e.g. Aurass et al. 2001) can indicate the presence of the coil-like (helical) magnetic field structure. Up to now, we have been speaking about a coil-like structure that has its axis more or less parallel to the density gradient. On the other hand if we assume a coil-like (or helical) structure having its axis perpendicular to the density gradient, then the electron beams can generate radio bursts with both positive and negative frequency drifts, but in a limited frequency range. Moreover, the resulting radio spectrum can be modified by the absorption, which in the $\mathrm{GHz}$ frequency range can be very high (Benz et al. 1992). Thus the absorption effects can visualize only parts of the electron beam trajectory and, for example, only the bursts generated in the observed side of the coil-like structure can be recorded. In this case, all bursts can have positive (or negative) frequency drifts. All these ideas give us new perspective in the interpretation of the radio spectra observed at the beginning of eruptive solar flares.

Acknowledgements. The authors thank K. Jiřička and H. Mészárosová for the technical assistance. This work was supported by the grants A3003202, S1003006 and A3003003 of the Academy of Sciences of the Czech Republic and by the grants 205/00/1727, 102/00/1711 of the Grant Agency of the CzechRepublic under the key project K2043105; and by the 
Project of Bilateral Cooperation (Germany - Czech Republic) in Science and Technology TSR-012-98 (ME 358). Some data used courtesy of the SOHO/EIT, MDI consortium. SOHOis joint ESA-NASA program.

\section{References}

Amari, T., Luciani, J. F., Mikic, Z., \& Linker, J. 2000, ApJ, 529, L49

Antiochos, S. K., DeVore, C. R., \& Klimchuk, J. A. 1999, ApJ, 510,485

Aurass, H., Shibasaki, K., Reiner, M., \& Karlický, M. 2001, ApJ, 567, 610

Ballester, J. L., \& Kleczek, J. 1983, Sol. Phys., 87, 261

Ballester, J. L., \& Kleczek, J. 1984, Sol. Phys., 90, 37

Bastian, T. S., Benz, A. O., \& Gary, D. E. 1998, ARA\&A, 36, 131

Benz, A. O. 1993, Plasma Astrophysics (Kluwer Academic Press, Dordrecht, The Netherlands), 89

Benz, A. O., Magun, A., Stehling, W., \& Su, H. 1992, Sol. Phys., 141, 335

Brown, J. C. 1971, Sol. Phys., 18, 489

Chen, J. 1996, J. Geophys. Res., 101, 27499

Ciaravella, A., Raymond, J. C., Thompson, B. J., et al. 2000, ApJ, 529, 575

Delaboudinière, J.-P., Artzner, G. E., Brunaud, J., et al. 1995, Sol. Phys., 162, 291

Dere, K. P., Brueckner, G. E., Howard, R. A., Michels, D. J., \& Delaboudinière, J.-P. 1999, ApJ, 516, 465

Hagyard, M. J. 1988, Sol. Phys., 115, 107

Hood, A. W., \& Priest, E. R. 1979, Sol. Phys., 64, 303
Hori, K. 2000, ApJ, 543, 1011

Karlický, M., Kotrč, P., \& Kupryakov, Y. A. 2001a, Sol. Phys., 199, 145

Karlický, M., Yan, Y., Fu, Q., et al. 2001b, A\&A, 369, 1104

Melrose, D. B. 1991, ApJ, 381, 306

Moore, R. L. 1988, ApJ, 324, 1132

Parker, E. N. 1974, ApJ, 191, 245

Plunkett, S. P., Vourlidas, A., Šimberová, S., et al. 2000, Sol. Phys., 194, 371

Priest, E. R., 1982, Solar Magnetohydrodynamics (D. Reidel Publ. Comp., Dordrecht, Holland), 78

Rompolt, B., \& Mzyk, S. 1978, in Proc. of the Colloq. IAU 44 Physics of Solar Prominences, ed. E. Jensen, P. Maltby, \& F. Q. Orrall, Inst. of Theoretical Astrophysics, Oslo, 242

Rompolt, B. 1990, Hvar Obs. Bull., 14, 37

Schmahl, E., \& Hildner, E. 1977, Sol. Phys., 55, 473

Schmieder, B., van Driel-Gesztelyi, L., Wiik, J. K., et al. 1997, Proc. of the Fifth SOHO Workshop, ESA SP-404, 663

Suzuki, S., \& Dulk, G. A. 1985, in Solar Radiophys. ed. D. J. McLean, \& N. R. Labrum (Cambridge University Press, Cambridge, UK)

Tandberg-Hanssen, E. 1974, Solar Prominence (D. Reidel Publ. Co., Dordrecht, Holland)

Tandberg-Hanssen, E. 1995, The Nature of Solar Prominences (Kluwer Acad. Publishers, Dordrecht, The Netherlands), 90

Vršnak, B. 1990, Sol. Phys., 129, 295

Vršnak, B., Ruždjak, V., \& Rompolt, B. 1991, Sol. Phys., 136, 151

Wang, Y. M., \& Sheeley, N. R. Jr. 1999, ApJ, 510, L157

Wood, B. E., Karovska, M., Chen, J., et al. 1999, ApJ, 512, 484 